J. Comp. Int. Sci. (2016) 7(1):03-11

http://epacis.net/jcis/PDF_JCIS/JCIS-0102.pdf

jcis@epacis.net

@2016 PACIS (http://epacis.net)

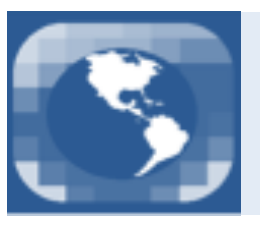

\title{
Routing metrics in wireless mesh networks: a Monte Carlo simulation approach
}

\author{
Marlon da Silva ${ }^{a},{ }^{1}$, Ana Clara Rodrigues Alves $^{b}$, Nandamudi Vijaykumar ${ }^{c}$, \\ Edson Luiz Frana Senne ${ }^{d}$ and Gerson da Penha Neto ${ }^{b}$ \\ ${ }^{a}$ Center for Monitoring and Early Warning of Natural Disasters, SJCampos, SP, Brazil \\ ${ }^{b}$ FATEC, SJCampos, SP, Brazil \\ ${ }^{c}$ National Institute for Space Research, SJCampos, SP, Brazil \\ ${ }^{d}$ UNESP, Guaratinguetá, SP, Brazil \\ Received on September 24, 2015 / accepted on January 29, 2016
}

\begin{abstract}
Wireless Mesh Networks (WMN) can extend the wireless technologies with respect to their coverage. In order to properly plan a WMN, it is not always easy to install it directly as the topology and configuration of some parameters may not be optimized leading to excessive costs and inefficient network services. Therefore, it is necessary to evaluate WMN performance with different configurations of parameters and different topologies without the need to deploy them. The paper presented here proposes the use of Monte Carlo simulation in order to evaluate the performance of a WMN in order to satisfy acceptable QoS parameters for routing metrics, such as ETX and ETT. Results presented show that the method is quite effective in testing several scenarios and configurations in order to obtain a good feasible solution before installing the network.
\end{abstract}

Keywords: Wireless Mesh Networks, WCETT, Simulation, Monte Carlo, Computational Mathematics.

\section{Introduction}

Wireless technology has offered important social changes due to its feasibility towards architectures and services and thus making a major contribution to its popularization. This technology is of low cost in the manufacturing of wireless devices, bringing an increase in the use of wireless devices, such as mobile phones, tablets and notebooks. As a matter of fact, many establishments such as shopping centers, airports, universities and public squares offer wireless access to Internet for many users that frequent such places. For example, several public sectors, such as States and Cities are offering free wireless access to their population. It is one of the approaches to promote digital inclusion. Wireless networks depend on APs (Access Points) that transmit and receive data, besides connecting the network to a wired network. The design of Wireless networks have to plan on how many Access Points are required in order to guarantee a significant area of coverage.

However, there are locations where it may not be quite simple to install wired Access Points and if the coverage area has to be expanded, a simple Wireless Network technology may not suffice. Therefore, Wireless Mesh Networks (WMNs) stand out as one of the main alternatives to expand wireless coverage areas [1]. Many institutions are adopting WMN to integrate different access points with installation and maintenance costs relatively low in relation to other types of wireless technology.

\footnotetext{
${ }^{1}$ E-Mail Corresponding Author: marlon.silva@cemaden.gov.br
} 
WMN is a network technology where some access points are interlinked by wireless connections, providing expansion of coverage area to remote locations without the need of extending wires for these connections. APs that compose a WMN are routers responsible to transmit data to other APs. This set of APs compose the backbone that provides the network coverage. However, only few APs are connected directly to external data source. These special devices of a WMN are known as gateways. Figure 1 presents an example of a WMN containing APs that compose the backbone.

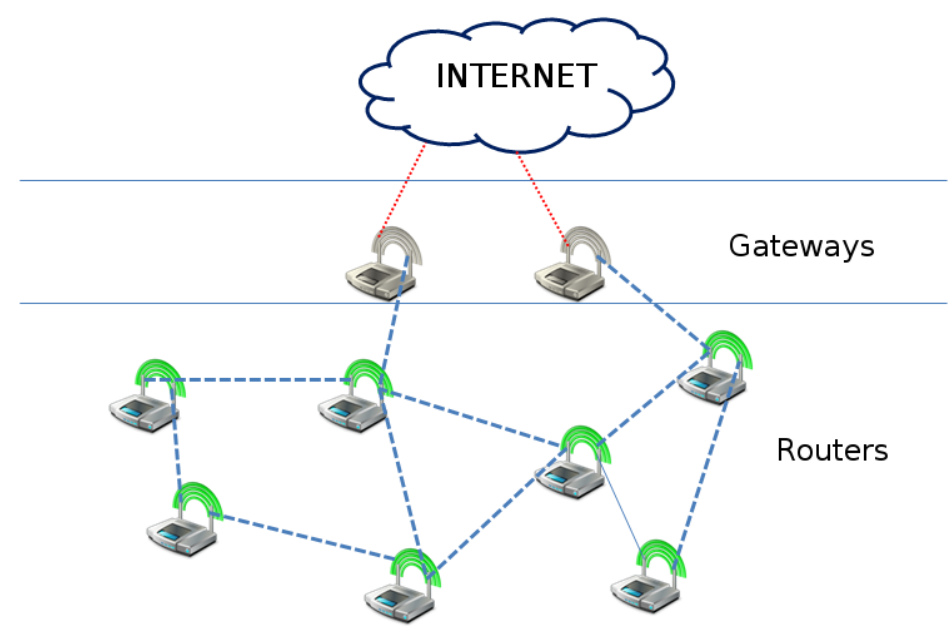

Figure 1: A wireless mesh network

In order to install a WMN, a proper planning is required to avoid problems that can affect the network performance [16]. Network performance may be evaluated based on some metrics that are used to quantify the quality levels for the network, such as packet loss, average delay, jitter, etc.

However, for WMNs, these metrics may not be adequate. Usually, for a packet to traverse from a source to its destination, it has to hop from an AP to another. So, these above mentioned metrics do not consider the time for such hops, i.e., the communication between APs. For this case, some other metrics are employed to quantify the quality of transmission within a hop, such as ETX (Expected Transmission Count) and ETT (Expected Transmission Time) [8]. These metrics consider the traverse that a data packet makes in a single hop, thus enabling a closer diagnosis on which hop may be affecting the quality of transmission of a packet.

The work discussed in this paper focuses on extracting metrics, based on Quality of Service (QoS) parameters of a WMN: loss of packets and quantification of number of hops (ETX and ETT). ETX (Expected Transmission Count) determines the average throughput of transmissions while ETT calculates the average time of transmission [14]. Based on these three metrics, it becomes possible to choose the best topology that satisfies the requirements for a given installation.

Now, the question is how to evaluate such metrics? The answer lies in employing, for example, simulation models. Based on the results, one can predict the behavior of a WMN and some of the parameters can be adjusted to improve the performance by adjusting the configuration, thus satisfying the client's requirements.

The objective of this paper is to simulate a WMN from a network configuration consisting of topology with a given number of APs and gateways. The proposed simulation model calculates the values, for some parameters, ETX and ETT, that are within the acceptable QoS standards. Simulation can reduce costs by analyzing the behavior of a WMN. The simulation model employed in this paper is Monte Carlo.

This paper contributes in presenting a simple alternative to simulate the behavior of a WMN and to obtain values for routing metrics used exclusively for this type of network. There are some simulators used for evaluating networks, such as Network Simulator 3 (NS-3) [7]. However, employing such simulator faces two issues that make their use somewhat difficult: routing metrics for WMNs can not be extracted directly from simulators and computational time is high when the network is of a relatively large size [17]. In this paper, the approach is to employ Monte Carlo simulation. The major advantage here is it is much more simple to be used and learned. Monte Carlo also needs to code the extraction of routing metrics, but, this 
is quite simple when compared to NS-3, because it does not need special libraries and moreover, can be installed on any platform.

The paper is organized as follows: Section 2 presents the metrics that are used to evaluate WMN as well as some published literature on Monte Carlo simulation. The proposed model is shown in Section 3 to extract ETX and ETT. Computational results are provided in Section 4 and finally Section 5 concludes the paper as well as pointing out some future directions.

\section{Background}

Before going through some published literature on the subject, it is essential to explain some of the terms that will be used throughout the paper $[1,11]$ :

- gateways: APs that receive directly data packets from external sources and provide them to the network;

- routers: APs that receive the signal from another AP and reply data packets to other routers or clients;

- clients: Customer devices of the network. Clients demand data packets to the WMN;

- links or hops: Wireless connections between two APs or between AP and client;

- protocol: System in which two network devices communicate via a common language;

- packet: Also known as network packet, it is a unit of data that is formatted and carried in the network;

- size of a packet: Total amount (usually in bytes) of formatted data contained in a packet;

- bandwidth: Refers to data transfer rate, i.e., amount of data that can be carried within a network. It is usually measured as bits per second.

WMNs have as a main feature multihops that refer to a path packet takes by hopping from one point to other until the required destination is reached. However, some even packets may not be handed over to final receiver. In this case, packet loss rate is used in order to measure how many packets are lost in the network [5]. Packet loss rate is denote by the ratio between the number of lost packets and the number of total packets in a network, according to all packets or a determined subset inside it.

So, some specific metrics have to be employed in order to understand more about the behavior of this traverse. It is important to note that the destination may have to be determined according to the routing protocols that are adopted as well as their respective strategies.

Due to many strategies of communication used by protocols, some traditional performance metrics may not be indicated to estimate how good is the transmission during the link. In addition to this, some metrics are very specific to evaluate link conditions on WMNs [15]. One such metric is ETX [12] that refers to the number of times an AP expects to transmit or retransmit a packet to a receiver without corrupting the packet during the delivery. ETX can be determined based on probability of packet loss during the request transmission $\left(p_{j}^{o}\right)$ and reception of response $\left(p_{j}^{d}\right)$ in a link $j$ :

$$
E T X_{j}=\frac{1}{\left(1-p_{j}^{o}\right)\left(1-p_{j}^{d}\right)}
$$

Another metric is ETT referring to the average time expected for each hop. So, this is used to evaluate how much time, on average, a packet takes to go through a link [3]. Given a link $j$, ETT is calculated in function of ETX $[8,10]$, size $\lambda_{j}$ of the packet and bandwidth $b_{j}$ by the following equation:

$$
E T T_{j}=E T X_{j} \frac{\lambda_{j}}{b_{j}}
$$

ETT, however, does not consider the communication problems within a link, such as interference caused between network channels that may have a consequence in assigning a new channel. In such cases, other 
metrics have to be investigated, which is not the scope of the paper. In fact, ETT evaluates just the average time spent for a single hop between two points. This gives an idea of the delay during a transmission in a network link. Both ETT and ETX are measured dynamically [12] and can be implemented in OLSR and AODV Protocol $[9,6]$.

Monte Carlo simulation is a method that can be employed as an alternative to predict, estimate and evaluate the behavior of a system [4]. This method uses values chosen randomly, according to some probability distribution previously defined. An example of application using Monte Carlo simulation is in [2] employed for emergency service system, where each server can be idle, busy attending either primary or secondary requests.

Monte Carlo simulation is recommended for systems where the number of servers is high, due to its quick response time to provide performance measures that allow best system evaluations in an acceptable computational time. Hence, Monte Carlo simulation can be an interesting tool and quite effective to obtain metrics for WMNs, such as rate of packet loss or throughput. As previously mentioned, rate of packet loss can be used as a parameter to obtain routing metrics for WMNs, such as ETX and ETT, where, the latter is a good estimative to understand the behavior of the transmission times.

\section{The Proposed Model}

The first step to build the model is the specification of network structure. The variables to be used in the model are important to represent the behavior of the network in runtime. Initially, two sets spatially distributed are defined. Let $\mathbf{P}=\{1,2, \ldots, n\}$ be the set of APs and $\mathbf{C}=\{1,2, \ldots, m\}$ the set of clients in fixed locations acting as test points. All elements of sets are represented in Cartesian coordinates.

The network traffic must consider demand rates from clients and service rates by APs. Demand rates can be approximated to Poisson process, while service rates can be modeled as an exponential distribution [2]. Each client has three different demand (or arrival) rates, each one representing a different type: simple data (denoted by $\lambda_{i}^{d}$ ), audio (denoted by $\lambda_{i}^{a}$ ) and video (denoted by $\lambda_{i}^{v}$ ), for all $i \in \mathbf{C}$. During the service to process (or attend) packets, audio data type and video data type have priority, in order to avoid decrease in the quality of multimedia transmissions..

Let $\mathbf{u}=\left(u_{1}, u_{2}, \ldots, u_{n}\right)$ be the vector that represents the state of APs. Each $u_{i}$ represents the number of data packets in the queue of AP $i \in \mathbf{C}$, i. e., when AP $i$ is idle, $u_{i}=0$ and, otherwise, $u_{i}>0$, if AP $i$ is busy. In this last case, data packet enters in the queue until a maximum capacity of queue, denoted by $Q$.

In order to avoid a significant computational effort, one of the strategies used to verify the quality of the system is Monte Carlo simulation [2] to evaluate environment behavior. However, some differences can be listed in our model: a server (AP) can service many atoms (clients) instead of only two; each client can demand three distinct types of rates, varying according to data type requested and one request, once demanded, enters in the queues of all APs that compose the path that data packet must travel to a client.

To simplify the algorithm, let $T$ be the variable that represents one of the data types that belong to this model - simple data $(d)$, audio $(a)$ and video $(v)$. Using these notations, Table 1 lists the parameters used in the algorithm and their respective descriptions.

Table 1: Variables used in the algorithm

\begin{tabular}{c|l}
\hline Variable & Description \\
\hline$\gamma_{T}^{[k]}$ & Number of data packets of type $T$ that enter network \\
$\gamma_{T, l o s s}^{[k]}$ & at instant $k$ \\
$\theta^{[k]}$ & Number of lost data packets of type $T$ in the network \\
$\mathbf{L}_{n}^{r}$ & Number of services up to instant $k$ \\
$\Lambda$ & Subset of links where $n$ is the receiver AP \\
\hline
\end{tabular}

Monte Carlo simulation proposed for WMNs consists of the following steps: 
1. Define initial values for

- $k=0$;

- $\mathbf{u}^{[k]}=(0,0,0, \ldots, 0)$;

- $\gamma_{T}^{[k]}=\gamma_{T, l o s s}^{[k]}=0$, for all $T \in\{d, a, v\}$;

- $\Theta^{[k]}=0$;

- $\Lambda=\sum_{T} \sum_{i=1}^{C} \lambda_{i}^{T}$;

2. Get a random value to $\omega \in[0,1]$;

3. If $\omega<\frac{\Lambda}{\Lambda+\Theta^{[k]}}$, then

- $\gamma_{T}^{[k+1]}=\gamma_{d}^{[k]}+1$, according to type $T$;

- Simulate randomly a client $\sigma \in \mathbf{C}$ and a type $T$ with probability $\lambda_{T \sigma} / \Lambda$;

- If $u_{\sigma}^{[k]}=0$ then $u_{\sigma}^{[k+1]}=1$;

- Else if $u_{\sigma}^{[k]}<Q$, then the request enters into queue, $u_{\sigma}^{[k+1]}=u_{\sigma}^{[k]}+1$ and, for all AP $\tau \in \mathbf{P}$ that composes the packet path, $u_{\tau}^{[k+1]}=u_{\tau}^{[k]}+1$;

- Else, the data packet is lost, $u_{\tau}^{[k+1]}=u_{\tau}^{[k]}$ and $\gamma_{T, l o s s}^{[k+1]}=\gamma_{T, l o s s}^{[k]}+1$;

4. If $\omega>\frac{\Lambda}{\Lambda+\Theta^{[k]}}$, then

- Simulate randomly an AP $\delta \in \mathbf{P}$ according to probability $\theta_{\delta}^{[k]} \Theta^{[k]}$, where:

$-\theta_{\delta}^{[k]}=0$ if $u_{\delta}^{[k]}=0$

$-\theta_{\delta}^{[k]}=\mu_{\delta}$ if $u_{\delta}^{[k]}>0 ;$

- $u_{\delta}^{[k+1]}=u_{\sigma}^{[k]}-1$;

5. If $u_{j}^{[k+1]}>0$, for all $j \in \mathbf{P}$, then $\Theta^{[k+1]}=\sum_{i: u_{i}>0} \mu_{i}$;

6. Repeat from Step 2 until a limited number $N$ of iterations.

After this iterative process, packet loss rate, denoted by $p_{T, l o s s}$, is calculated from the following equation:

$$
p_{T, \text { loss }}=\frac{\gamma_{T, l o s s}^{[N]}}{\gamma_{T}^{[N]}} .
$$

The next step of the algorithm is to calculate routing metrics. In this case, the first metric to be calculated is ETX for each data type. From Equation (3), ETX is obtained from loss rate $p_{T, l o s s}$. Hence, ETX can be estimated by:

$$
X_{T}=\frac{1}{\left(1-p_{T, l o s s}\right)^{2}} .
$$

This equation considers only the probability of loss in terms of packets. As ETX calculates distinct rates for sending and receiving data [8], then probability of success is multiplied twice.

To calculate ETT over network links for each data type, let $t_{T}$ be estimated ETT for a given type $T$. Considering demand (or arrival) rate of clients as transmission rate and service rate as bandwidth, ETT can be calculated as follows for all the clients for that specific AP:

$$
t_{T}=X_{T} \frac{\sum_{T} \lambda_{i}^{T}}{\sum \mu} .
$$




\section{Numerical Results}

The algorithm was tested on instances representing WMNs in a random topology in an outdoor environment, given the distance between neighbor APs is greater than 100 meters. The algorithm was built using $\mathrm{C}++$ and run in a computer with quad-core $\mathrm{CPU}$ of $2.5 \mathrm{GHz}$ and 4 GB RAM.

In the conducted tests, all APs have similar features, using IEEE 802.11 standard and the same buffer capacity of 300 data packets. Each AP has a maximum bandwidth capacity of $\mu_{j}=54 \mathrm{Mb} / \mathrm{s}$, and the sum of all rates, randomly distributed, per client is $\lambda_{i}^{a}+\lambda_{i}^{v}+\lambda_{i}^{d}=2 \mathrm{Mb} / \mathrm{s}$.

\subsection{Scenario 1}

The first scenario, located at São José dos Campos, Brazil, to be tested has 80 clients and 8 APs, with 2 gateways and 6 routers. Figure 1 shows the WMN topology of this scenario, where cyan points denote router, blue point is the gateway and the crosses are clients that are randomly distributed.

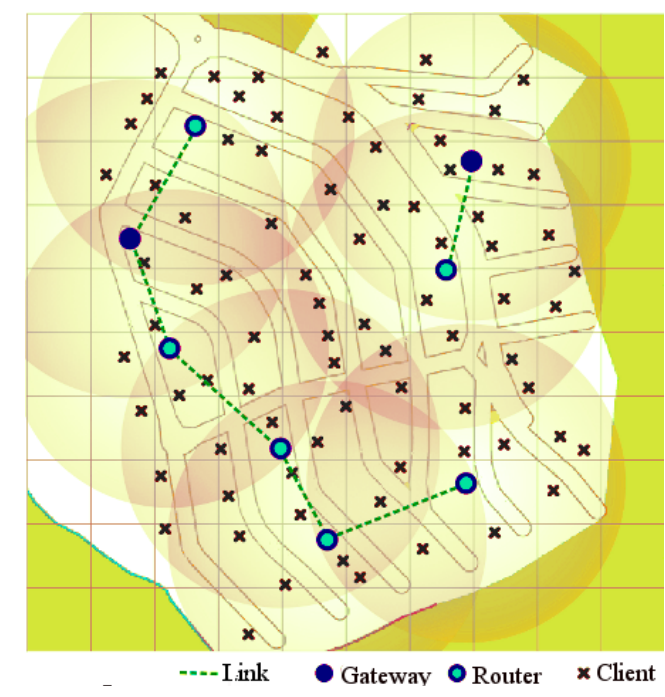

Figure 2: WMN used for Scenario 1

In the context of QoS, tolerable values for packet loss rate is $10 \%$ for video, $10 \%$ for audio and $10 \%$ for simple data. Hence, based on the given packet loss rate, for each data type, acceptable (or ideal) values for ETX are 1.2346. For comparison purposes, acceptable average delay for this case is $150 \mathrm{~ms}$ for video and 150 ms for audio packets (for more details about these values, see [18]). These values will be used to compare ETTs.

Monte Carlo simulation has been run 1000 times and the total computational time was 132 seconds. Considering confidence interval of 0.95 for results, average values for loss rate, ETX and ETT for each data type are described in Table 2.

Table 2: Results obtained for routing metrics for Scenario 1

\begin{tabular}{c|c|c|c|c}
\hline Variable & Description & Simple data & Audio & Video \\
\hline$p_{\text {loss }}$ & Loss rate & 0.1212 & 0.0699 & 0.0654 \\
$X$ & ETX & 1.2949 & 1.1560 & 1.1448 \\
$\tau$ & ETT $(\mathrm{ms})$ & 0.1918 & 0.1713 & 0.1696 \\
\hline
\end{tabular}

Based on the results from Table 2, the values of ETX obtained for audio and video data are less than accepted QoS levels (1.2346). On the other hand, ETX for simple data are not satisfied, when compared to acceptable levels. However, the values for ETT obtained for audio (171.3 ms) and video (169.6 ms) are higher than agreed values. In this case, the network infrastructure needs improvements, such as a better distribution of APs in order to reduce transmission time. 


\subsection{Scenario 2}

Another scenario used in [13], located at Pindamonhangaba, Brazil, to be tested has 150 clients and 13 APs, with 4 gateways and 9 routers. Figure 3 illustrates the topology of WMN. In this Figure, orange points denote gateways and yellow points represent routers.

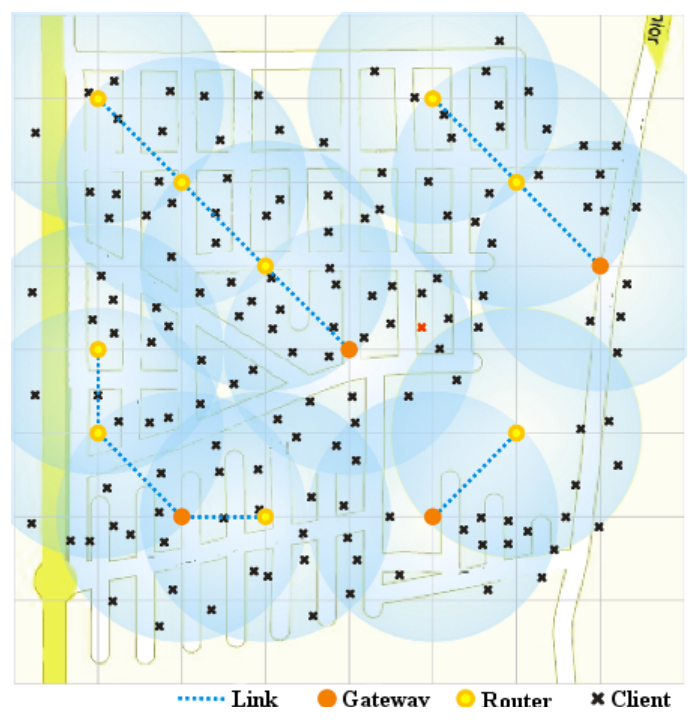

Figure 3: WMN used for Scenario 2 [13]

In this scenario, the same input parameters of Scenario 1 were used and the accepted values for ETX are the same as for Scenario 1.

Monte Carlo simulation has been run 1000 times and the total computational time was 132 seconds. Considering confidence interval of 0.95 for results, average values for loss rate, ETX and ETT for each data type are described in Table 3.

Table 3: Results obtained for routing metrics for Scenario 2

\begin{tabular}{c|c|c|c|c}
\hline Variable & Description & Simple data & Audio & Video \\
\hline$p_{\text {loss }}$ & Loss rate & 0.0759 & 0.0167 & 0.0162 \\
$X$ & ETX & 1.1710 & 1.0343 & 1.0332 \\
$\tau$ & ETT $(\mathrm{ms})$ & 0.1735 & 0.1532 & 0.1531 \\
\hline
\end{tabular}

Based on the results shown in Table 3, the values of ETX obtained for all types are less than QoS level for ETX (1.2346). Hence, all ETX that were obtained are within acceptable QoS levels. However, the values for ETT obtained for audio (153.2 ms) and video $(153.1 \mathrm{~ms})$ are slightly higher than agreed values, but as they are not that different, they may be considered satisfying as QoS parameters.

\section{Conclusions}

The work presented in this paper deals with a proper planning of Wireless Mesh Neworks (WMN) in order to satisfy QoS (Quality of Service) parameters. An algorithm was developed to verify QoS parameters, such as, packet loss and delay by means of Monte Carlo simulation. If the results of the simulation show that the designed topology does not satisfy QoS parameters, new restrictions are included and this process is repeated until an optimal topology that satisfies QoS is reached.

By employing algorithms to plan WMN, the main advantage is to understand the performance behavior of the network in a given location, without the need to deploy it. Based on the studies and consequent results, it is possible to minimize the costs and at the same time maintain that the network be more efficient in delivering the required services. So, evaluation of QoS parameters is extremely essential so that the deployed network is efficient by satisfying the clients' requests. 
The algorithm for planning a WMN, proposed in this paper, looks into solving Quality of Service offered by the network. This is extremely important as on one hand, all the clients of the network (WMN) must be within the coverage area so that they are able to receive the network signal. On the other hand, the requested packets (by the clients) must be received without loss and within a proper time limit without delays so that a multimedia transmission, for example, may not be affected.

Monte Carlo simulation has been very effective in calculating QoS parameters for a WMN in the tests presented in this paper, although these type of instances do not follow a defined sample, only random positions or previously proposed by geographic conditions, as in both scenarios. Consequently, these parameters may be used to design a real network. However, realistic scenarios face several issues, such as interferences from the channels, other equipment installed within the coverage area as well as natural phenomena.

For future works, simulation can include all these restrictions in order to understand the behavior of the network and, based on results, some of the parameters may be tuned in order (in an iterative process) to get the best configuration and topology as well as minimization of costs.

Acknowledgments: The first two authors duly acknowledge CNPq for the Ph.D. and PIBIC fellowships respectively, and INPE/LAC providing infrastructure and necessary support to develop this work. .

\section{References}

[1] I. F. Akyildiz, X. Wang, and W. Wang, "Wireless mesh networks: a survey," Computer Networks ISDN Systems, vol. 47, no. 4, pp. 445-487, mar. 2005. [Online]. Available: http://www.sciencedirect.com/science/article/pii/S1389128604003457

[2] J. Atkinson, I. Kovalenko, N. Kuznetsov, and K. Mykhalevych, "A hypercube queueing loss model with customer-dependent service rates," European Journal of Operational Research, vol. 191, no. 1, pp. 223 - 239, 2008. [Online]. Available: http://www.sciencedirect.com/science/article/pii/S0377221707008855

[3] V. C. M. Borges, M. Curado, and E. Monteiro, "A cross-layer routing scheme for scalable triple play service in wireless mesh networks," in Computer Communications and Networks (ICCCN), 2010 Proceedings of 19th International Conference on, aug. 2010, pp. 1-6.

[4] P. Brémaud, Markov chains: Gibbs fields, Monte Carlo simulation, and queues. Springer Science \& Business Media, 2013, vol. 31.

[5] M. Buddhikot, S. Miller, and A. Subramanian, "Interference aware routing in multi-radio wireless mesh networks," 2013, uS Patent 8,532,023. [Online]. Available: https://www.google.com/patents/US8532023

[6] M. Campista, P. Esposito, I. Moraes, L. Costa, O. Duarte, D. Passos, C. Albuquerque, D. Saade, and M. Rubinstein, "Routing metrics and protocols for wireless mesh networks," Network, IEEE, vol. 22, no. 1, pp. $6-12$, jan.-feb. 2008.

[7] G. Carneiro, P. Fortuna, and M. Ricardo, "Flowmonitor: A network monitoring framework for the network simulator 3 (ns-3)," in Proceedings of the Fourth International ICST Conference on Performance Evaluation Methodologies and Tools, ser. VALUETOOLS '09. ICST, Brussels, Belgium, Belgium: ICST (Institute for Computer Sciences, Social-Informatics and Telecommunications Engineering), 2009, pp. 1:1-1:10. [Online]. Available: http://dx.doi.org/10.4108/ICST.VALUETOOLS2009.7493

[8] R. Draves, J. Padhye, and B. Zill, "Routing in multi-radio, multi-hop wireless mesh networks," in Proceedings of the 10th annual international conference on Mobile computing and networking, ser. MobiCom '04. New York, NY, USA: ACM, 2004, pp. 114-128. [Online]. Available: http://doi.acm.org/10.1145/1023720.1023732

[9] P. Esposito, M. Campista, I. Moraes, L. Costa, O. Duarte, and M. Rubinstein, "Implementing the expected transmission time metric for olsr wireless mesh networks," in Wireless Days, 2008. WD '08. 1st IFIP, nov. 2008, pp. 1-5. 
[10] M. Genetzakis and V. Siris, "A contention-aware routing metric for multi-rate multi-radio mesh networks," in Sensor, Mesh and Ad Hoc Communications and Networks, 2008. SECON '08. 5th Annual IEEE Communications Society Conference on, june 2008, pp. $242-250$.

[11] A. Khreishah, C.-C. Wang, and N. Shroff, "Cross-layer optimization for wireless multihop networks with pairwise intersession network coding," Selected Areas in Communications, IEEE Journal on, vol. 27, no. 5, pp. 606-621, 2009.

[12] A. Lavén and P. Hjärtquist, "Multimetric olsr and ett," in 5th OLSR Interop Ė Workshop, 2009.

[13] V. d. A. Machado, C. M. Hirata, M. d. Silva, N. L. Vijaykumar, C. N. Silva, R. d. S. Oliveira, C. R. L. Francês, J. C. W. A. Costa, and E. L. F. Senne, "Uma abordagem para garantia de qualidade em redes sem fio em malha," in Anais... Simpósio Brasileiro de Pesquisa Operacional, 44. (SBPO) e Congresso Latino-Iberoamericano de Investigación Operativa, 16 (CLAIO)., 2012. [Online]. Available: http://urlib.net/dpi.inpe.br/plutao/2012/11.28.01.53.54

[14] D. Passos, C. de Albuquerque, M. Campista, L. Costa, and O. Duarte, "Minimum loss multiplicative routing metrics for wireless mesh networks," Journal of Internet Services and Applications, vol. 1, no. 3, pp. 201-214, 2011. [Online]. Available: http://dx.doi.org/10.1007/s13174-010-0015-6

[15] B. Pinheiro, V. Nascimento, R. Lopes, E. Cerqueira, and A. Abelem, "A fuzzy queue-aware routing approach for wireless mesh networks," Multimedia Tools and Applications, vol. 61, pp. 747-768, 2012. [Online]. Available: http://dx.doi.org/10.1007/s11042-011-0933-8

[16] M. Silva, E. L. F. Senne, and N. L. Vijaykumar, "Wireless mesh networks planning based on parameters of quality of service," in Proceedings of 1st International Conference on Operations Research and Enterprise Systems, vol. 1, Vilamoura, 2012, pp. 441-446. [Online]. Available: http://dx.doi.org/10.5220/0003760104410446

[17] M. d. Silva, "Novo modelo de minimização de tempos de transmissão fim a fim em redes sem fio em malha," Ph.D. dissertation, Instituto Nacional de Pesquisas Espaciais (INPE), São José dos Campos, 2015-03-30 2015. [Online]. Available: http://urlib.net/sid.inpe.br/mtc-m21b/2015/03.11.14.56

[18] P. Wang and W. Zhuang, "A collision-free mac scheme for multimedia wireless mesh backbone," Wireless Communications, IEEE Transactions on, vol. 8, no. 7, pp. 3577-3589, July 2009. 